\title{
IDENTIFICACIÓN DE ÁRBOLES POTENCIALES DE (THEOBROMA CACAO L.) EN SIUNA Y ROSITA, JUNIO 2009 - JUNIO 2010
}

Oscar Montalván Castellón ${ }^{[1]}$

\section{Resumen}

La investigación se orientó a la selección de árboles potenciales de cacao, caracterizando los ambientes para los cuales se describió el comportamiento productivo de los árboles potenciales y los resistentes a plagas y enfermedades. Se estudiaron 15 unidades de producción, 8 en la comunidad el Carao, dos en el Guayabo, dos en Rosa Grande y una en Floripón del municipio de Siuna, una en el Zopilote y una en Santa Rita del municipio de Rosita. Esta investigación servirá como una herramienta base para nuevos estudios y un instrumento para el mejoramiento del cultivo del cacao a nivel municipal y regional, donde sean beneficiarios los productores inmersos en el proceso de mejoramiento del cultivo. Se identificó un total de 78 árboles potenciales, y se caracterizaron los ambientes, comportamiento productivo, resistencia a plaga $y$ enfermedades, características agroecológicas y morfológicas.

Entre los principales resultados sobresale la producción promedio por árbol, este se comportó de la manera siguiente: Entre 3.11 a 4.6 kilogramos por árbol en los tipos angoletas, 3.1 a 4.00 kilogramos en los amelonados, 3.11 a 4.7 en los cundeamor y o.6 kilogramos en los calabacín. La mayoría de los árboles evaluados presentaron afectaciones por plagas y enfermedades como: Moniliasis, mazorca negra, Cherelle wild y gusanos en los frutos, así como afectaciones por tandas en ramas.

Palabras clave: Amelonado, Angoleta, Cundeamor, Calabacín, Moniliasis, Phytoptora, Cherelle wild.

\section{Summary}

The research focused on the selection of potential cacao trees, characterizing the environments that describes the productive behaviour of these trees and those that are resistant to plagues and diseases. 15 productive units were analized, distribuited as followed: 8 in the community of Carao, 2 in Guayabo, 2 in Rosa Grande, 1 in Floripon -municipality of Siuna-, 1 in Zopilote and 1 in Santa Rosa -municipality of Rosita-. This research will serve as a tool for the development of other researches, and as an instrument to improve the cacao production at a municipal and regional

[1] Ingeniero agroforestal. Msc. Docencia universitaria. Técnico investigador de la URACCAN. Email. montesiuna@yahoo.com 
level, where the direct beneficiaries will be the producers who are involved in the process of crop improvement. A total of 78 potential cacao trees were identified, and the environments were characterized, also the productive behaviour, the resistance to plagues and diseases, as well as the agroecological and morphological characteristics.

Among the main results it's important to mention the prevalence of the average production per tree, which behaved as follows: between 3.11 to $4.6 \mathrm{~kg}$ per tree in angoletas type, 3.1 to $4.00 \mathrm{~kg}$ in amelonados, 3.11 to $4.7 \mathrm{~kg}$ in cundeamor and $0.6 \mathrm{~kg}$ in the calabacin type. Most trees presented damages by plagues and diseases such as: Moniliasis, black-cob, wild Cherelle and worms in the fruits, as well as branches affectations.

Keywords: Amelonado, angoleta, Cundeamor, Zucchini, Moniliasis, Phytoptora, wild Cherelle.

\section{Introducción}

El cultivo de cacao (Theobroma cacao L.) es un rubro de importancia económica en Nicaragua. De este cultivo dependen muchas familias al poder generar empleos e incrementar los ingresos. Cuando se desarrollan nuevos cultivares (clones), bien sea por selección, hibridación u otros métodos de mejoramiento genético, las instituciones científicas necesitan difundir los atributos agronómicos mejorados de estos nuevos materiales genéticos. Sin embargo, esta información en la mayoría de los casos no llega a los municipios donde los productores están cultivando cacao.

Se está estableciendo este cultivo en extensiones considerables en los municipios de Siuna y Rosita por comunitarios que se encuentran en zonas de amortiguamiento de la Reserva de Biosfera (Bosawás), y otros que han visto en este cultivo una de las formas para obtener ingresos, sin causar deterioro severo en el medio ambiente. Las variedades que se cultivan se han obtenido de híbridos traídos al municipio de centros experimentales de Honduras (FHIA) y Nicaragua (centro experimental El Recreo, y el Rama, RAAS), desde hace aproximadamente tres décadas, estas plantaciones se han utilizado como semilleros sin la selección de árboles élites (superiores) específicos que garanticen buena productividad, calidad de granos y resistencia a plagas y enfermedades.

Por tal razón, se lleva a cabo una selección de tipos de cacao para determinar rendimientos productivos, resistencia a plagas y enfermedades, y mediante pruebas de laboratorio los contenidos de antioxidantes, proteínas, grasas y características 
organolépticas. La investigación se realiza en los municipios de Siuna y Rosita específicamente en las comunidades que tienen plantaciones de cacao mayores de 10 años (Carao, el Guayabo, Floripón, el Zopilote y Santa Rita).

La investigación se enfoca en la selección de árboles potenciales de cacao donde se caracterizaron los ambientes donde se encuentran las plantaciones de cacao, se describió el comportamiento productivo de los árboles potenciales y los resistentes a plagas y enfermedades.

\section{Revisión de literatura}

El cacao es originario de las selvas del Amazonas. Los aztecas y los mayas lo cultivaban mucho antes del descubrimiento de América y lo consideraban de origen divino. El nombre del género es Theobroma, que significa alimento de los dioses. Comprende más de 25 especies diferentes; pero solo una, Theobroma Cacao L. se cultiva comercialmente. En la actualidad se encuentra ampliamente distribuida en los países tropicales, entre $\operatorname{los} 18^{\circ}$ de latitud norte y $15^{\circ}$ de latitud sur y una altura comprendida entre 300 a 700 msnm (Sánchez, 1990, p. 11).

El árbol de cacao es de tamaño mediano, aunque puede alcanzar altura hasta 20 metros cuando crece libremente bajo sombra intensa. Tiene un tronco recto que se puede desarrollar en forma muy variado, según las condiciones ambientales. Por lo general, el cacao proveniente de semilla, que crece normalmente, tiene su primer molinillo u horqueta entre 80 y $120 \mathrm{~cm}$; en ese puente nace un piso con tres a seis ramas principales que forman el árbol (Calderón, 1935, p. 9).

\section{Plantaciones establecidas con árboles potenciales de alta producción}

La selección de los árboles superiores de cacao debe ser hecha por cada familia ya que son ellas las que mejor conocen sus árboles de cacao. La evaluación de los árboles superiores se hará con la guía y orientación de promotores y técnicos. Cada familia deberá designar por lo menos a un miembro de su familia (responsable) para que haga las evaluaciones y registros de datos, según las indicaciones de los promotores (Cerda p.1-3).

\section{Comportamiento productivo de los árboles potenciales de cacao}

El injerto es el método más generalizado de reproducción vegetativa del cacao; se recurre a esta práctica cuando se desea reproducir fielmente las características de los árboles que se han seleccionado, evitando así la variación sobre todo en el comportamiento productivo, que normalmente ocurre con la propagación por semillas (Bacardit, 2001, p.1). 


\section{Relación entre el promedio y el máximo número de semillas por fruto}

Esta relación se determina de la misma muestra usada para las medidas de los dos descriptores componentes (Avilés, 2008, P6).

\section{Caracterización ambiental de plantaciones de cacao}

El crecimiento, desarrollo y buena producción del cacao están estrechamente relacionados con las condiciones medioambientales de la zona donde se cultiva. Es por ello que los factores climáticos influyen en la producción de plantaciones; por lo tanto las condiciones térmicas y humedad deben ser satisfactorias para el cultivo por ser una planta perenne y su período vegetativo en época de floración, brotamiento y cosecha, está regulado por el clima, cuya relación en el transcurso climático y el período vegetativo nos permite establecer los calendarios agroclimáticos (PROAMAZONIA, 2004, p. 9).

\section{Plagas y enfermedades}

El cacao es una de las plantas económicas que, al mismo tiempo que pueden sufrir daños considerables a causa de los insectos, también necesita de algunos de ellos en ciertos procesos reproductivos; por ello, un abuso en el uso indiscriminado de insecticidas puede conducir a posteriores fracasos económicos. Las enfermedades más comunes son: La mazorca negra, Mal del machete, Las bubas, La Moniliasis, Cherelle wild (Enríquez s/f, p.8).

\section{Materiales y métodos}

La investigación es de tipo cuantitativa, y en ella se utilizó la técnica de registro estructurado de observación para el levantamiento de la información sobre los componentes principales de las características agronómicas en árboles élites de cacao, así como caracterización morfo-agronómica y comportamiento de los mismos. Para el procesamiento de los datos se utilizó el software o programa Stastical Package for the Social Sciences.

\section{Resultados y discusión}

\section{Descripción general del estudio}

Los municipios de Siuna y Rosita presentan características similares para el establecimiento de cacao (Theobroma cacao), precipitaciones de 2000-2500 milímetros al año, temperaturas media anual entre 24-26 grados, alturas sobre el nivel del mar, 05 a 480 y pendientes de 2 a 38\%, también presentan suelos de texturas francos a franco arcillosas, idóneas para la implementación de este cultivo. Esta investigación se 
realizó en cinco comunidades que se dedican al cultivo de cacao: El Carao, El Guayabo y Floripón en el municipio de Siuna. El Zopilote y Santa Rita en el municipio de Rosita.

\section{Caracterización de los ambientes donde se encuentran las plantaciones de cacao}

Para identificar las condiciones ambientales sobre los índices productivos se estudiaron las siguientes características: precipitación, temperatura, altura sobre el nivel del mar, pendiente y porcentajes de sombreamiento.

Cuadro No. 1. Condiciones agroecológicas de las comunidades donde se realizó la investigación

\begin{tabular}{|l|c|c|c|c|c|}
\hline Comunidad & $\begin{array}{c}\text { Precipitación } \\
\text { anual } \\
\text { (milímetros) }\end{array}$ & $\begin{array}{c}\text { Temperatura } \\
\text { media anual } \\
\text { (grados } \\
\text { centígrados) }\end{array}$ & $\begin{array}{c}\text { Altura sobre } \\
\text { el nivel del } \\
\text { mar (msnm) }\end{array}$ & $\begin{array}{c}\text { Pendiente } \\
\text { (\%) }\end{array}$ & $\begin{array}{c}\text { Porcentaje de } \\
\text { sombra de los } \\
\text { cacaotales. } \\
\text { (\%) }\end{array}$ \\
\hline Carao & $2000-2500$ & $24-26$ & $150-253$ & $2-15$ & $25-40$ \\
\hline Guayabo & $2000-2500$ & $24-26$ & $464-525$ & $12-24$ & $30-32$ \\
\hline Floripón & $2000-2500$ & $24-26$ & $105-110$ & $2-4$ & $20-22$ \\
\hline Zopilote & $2000-2500$ & $24-26$ & $86-95$ & $5-9$ & $25-36$ \\
\hline Santa Rita & $2000-2500$ & $24-26$ & $55-68$ & $3-5$ & $30-35$ \\
\hline
\end{tabular}

La temperatura y precipitación no tienen variaciones significativas en dichas comunidades ya que los parámetros se dan de forma global por ser una región del trópico húmedo muy extensa, además, el distanciamiento de una comunidad a otra no sobrepasa los 100 kilómetros. La altura sobre el nivel del mar tiene variaciones en plantaciones del municipio de Rosita que se encuentra más cercana a la costa marítima del Atlántico, en cambio en plantaciones de la comunidad el Guayabo del municipio de Siuna las altitudes son superiores. Los porcentajes de pendientes son mayores a medida que la altura sobre el nivel del mar se incrementa. Astorga $C$ y A. Mora, afirman que los suelos deben presentar pendientes considerables (máximo 40\%), o que tengan un buen drenaje o sean fáciles de drenar con la construcción de canales. Los porcentajes de sombreamiento en las áreas en estudio oscilaron entre 20 y $40 \%$, estos porcentajes tienen una relación acorde al manejo que el productor realiza (Cuadro 1).

\section{Características morfológicas de los árboles seleccionados por tipo y por comunidad}

Se identificaron algunas características sobresalientes como: altura del árbol, diámetro de copa, número de ramas, cojines florales diámetro del tronco a $30 \mathrm{~cm}$. Estos datos nos dan parámetros para determinar la influencia de las condiciones ambientales en la producción de las plantaciones. 


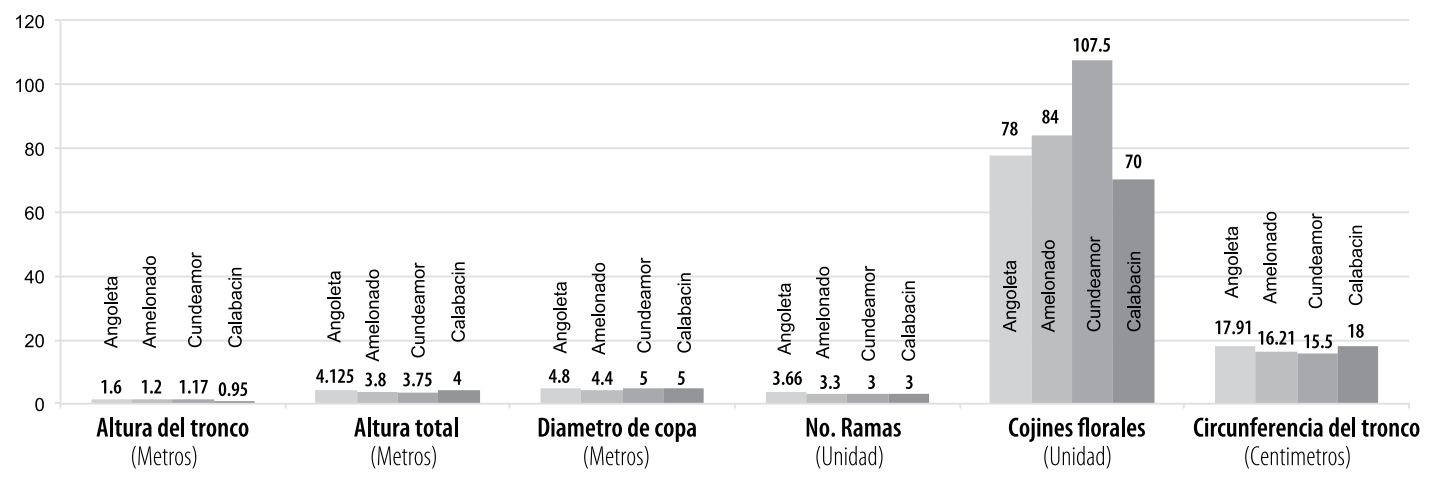

Figura No. 1. Descriptores morfológicas de árboles seleccionados en la comunidad El Carao.

En la comunidad el Carao se lograron muestrear 24 árboles con frutos forma angoleta, 23 con frutos amelonados, 2 con frutos cundeamor y 1 fruto calabacín. Los datos morfológicos de altura del tronco a la primera horqueta están dados de 0.95 a 1.6 metros, la altura total 3.75 a 4.125 metros, el diámetro de copa de 4.4 a 5 metros, número promedio de ramas de 3, cojines florales oscilaron desde 45 como mínimo hasta 141, la circunferencia del tronco osciló entre 11 a 25 centímetros.

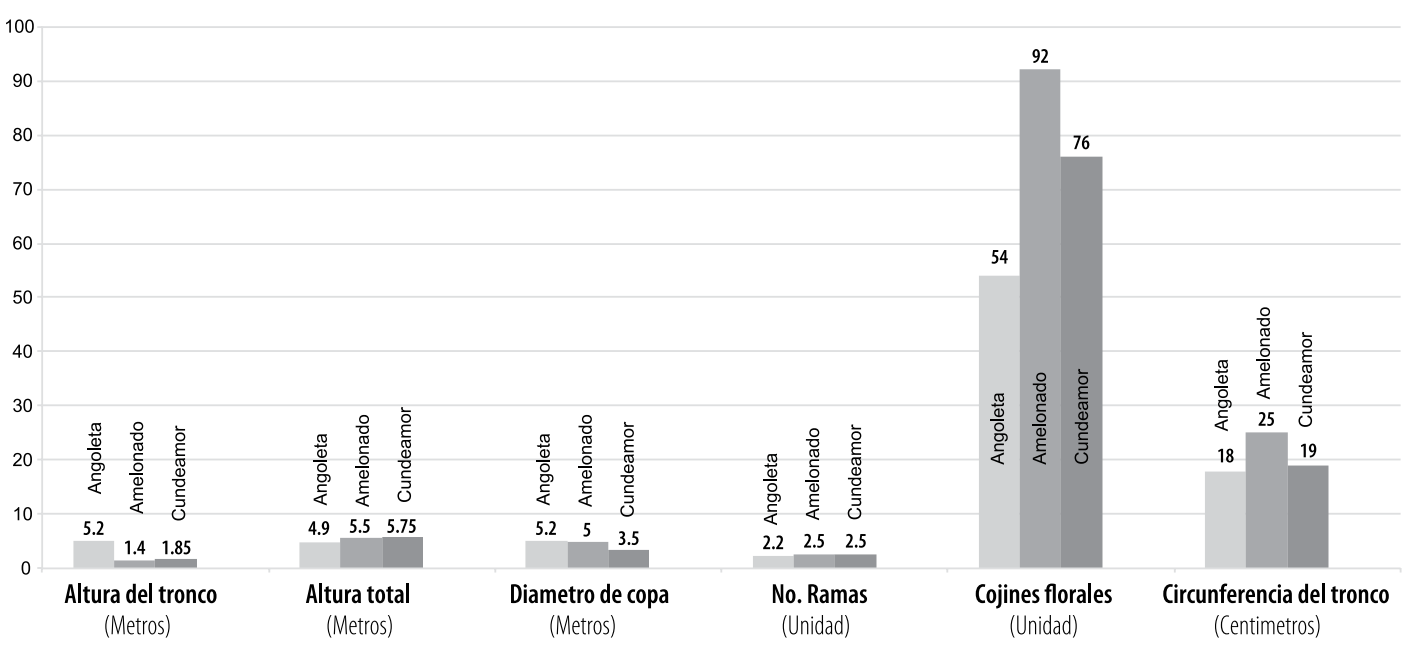

Figura No. 2. Descriptores morfológicos de árboles seleccionados en la comunidad El Floripón.

En la comunidad El Floripón se muestrearon 5 árboles con frutos forma angoleta, 2 con frutos amelonados, 2 con frutos cundeamor. Los datos morfológicos de altura del tronco fueron de 1.4 a 5.2 metros, la altura total 4.9 a 5.75 metros, diámetro de copa de 3.5 a 5.2 metros, número promedio de ramas de 2-3, cojines florales, desde 41 hasta 132, circunferencia del tronco 18 a 25 centímetros. 


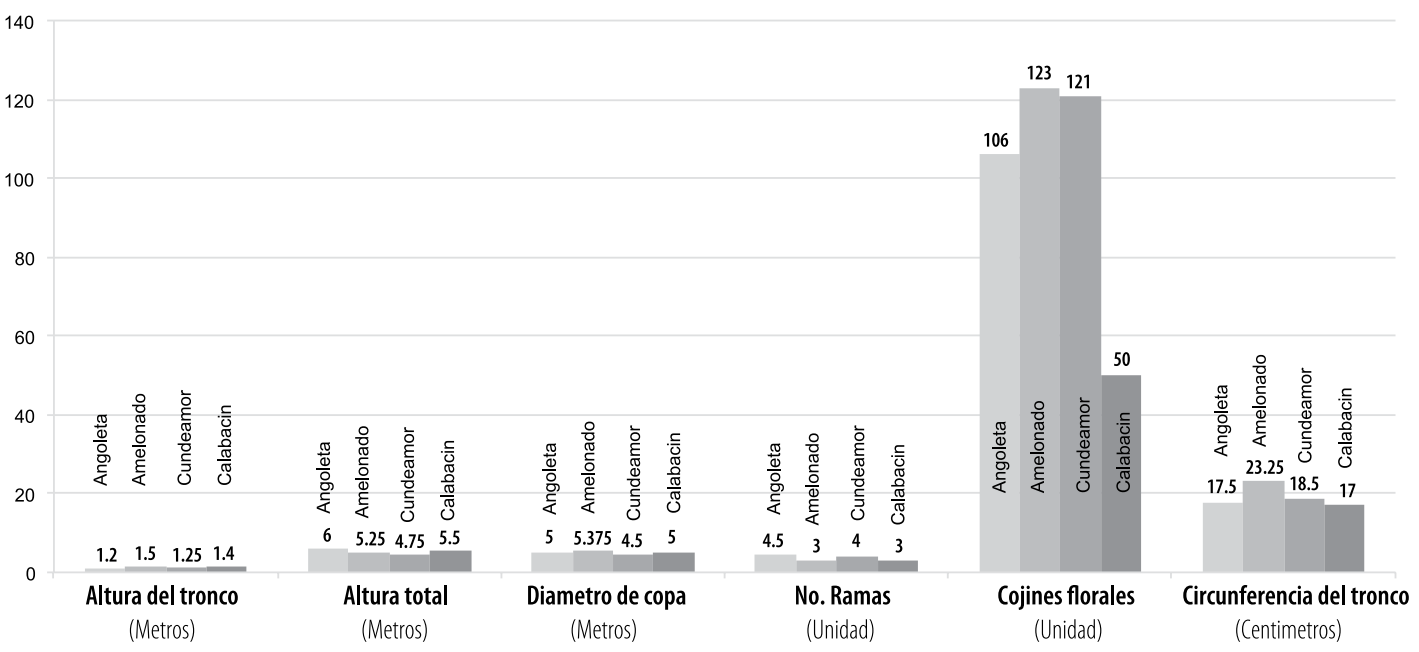

Figura No. 3. Descriptores morfológicos de árboles seleccionados en la comunidad El Guayabo.

En la comunidad El Guayabo se muestrearon 2 árboles con frutos forma angoleta, 4 con frutos amelonados, 2 con frutos cundeamor, 1 árboles tipo calabacín. Los datos morfológicos de altura del tronco a la primera horqueta fueron de 1.2 a 1.5 metros, la altura total de los árboles fue de 4.0 a 6.0 metros, el diámetro de copa de 4.0 a 5.0 metros, número promedio de ramas de 3.0 - 4.0, los cojines florales oscilaron desde 50 como mínimo hasta 200, presentado por los tipos amelonados, la circunferencia del tronco osciló entre 17 a 30 centímetros.

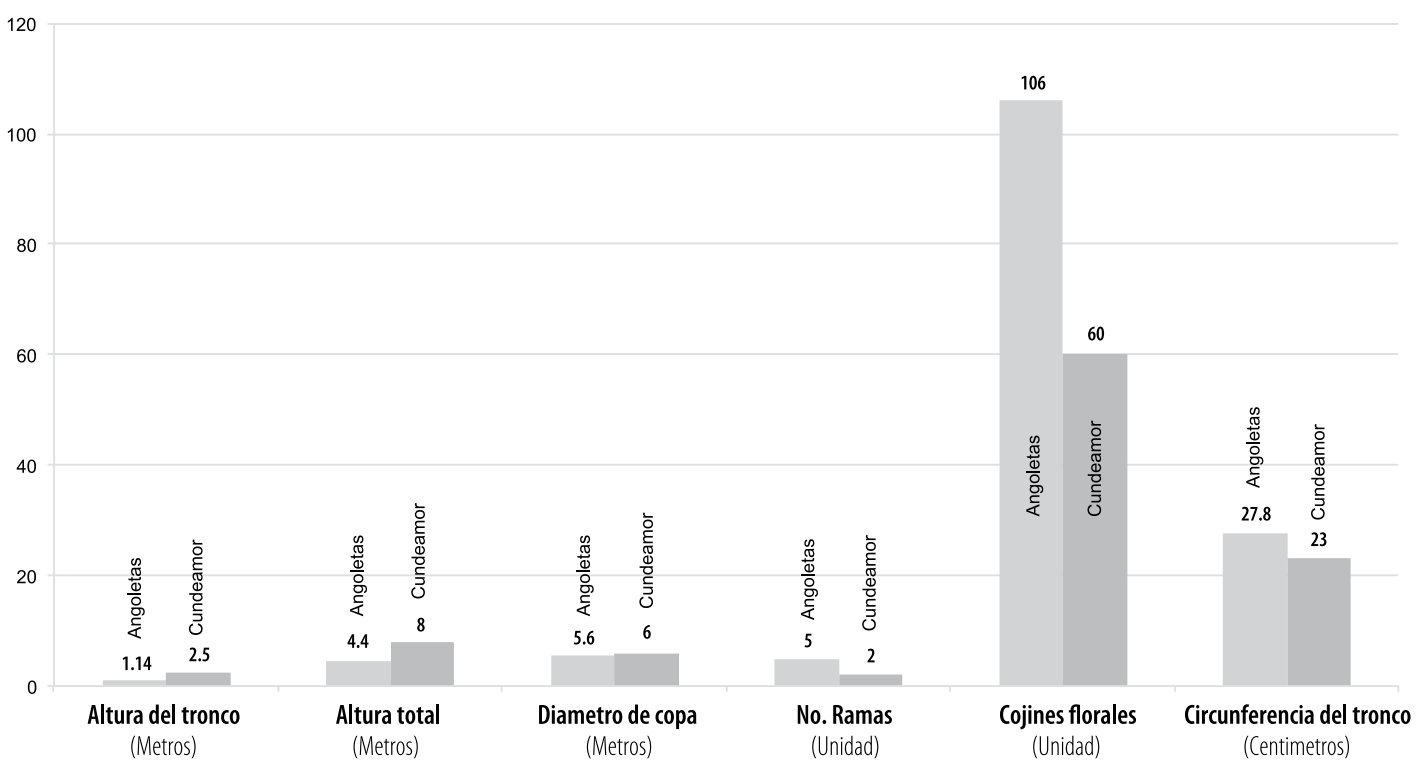

Figura No. 4. Descriptores morfológicos de árboles seleccionados en la comunidad Santa Rita. 
La comunidad de Santa Rita pertenece al municipio de Rosita, se evaluaron 5 árboles con frutos de forma angoleta, 1 con frutos cundeamor. Los datos morfológicos de altura del tronco a la primera horqueta fueron de o.6 - 2.5 metros, la altura total de los árboles fue de 4.0 a 8.0 metros, el diámetro de copa de 4.0 a 6.0 metros, número promedio de ramas de 2.0 - 4.0, los cojines florales oscilaron desde 60 como mínimo hasta 150, presentado por los tipos amelonados, la circunferencia del tronco osciló entre 20 a 26 centímetros.

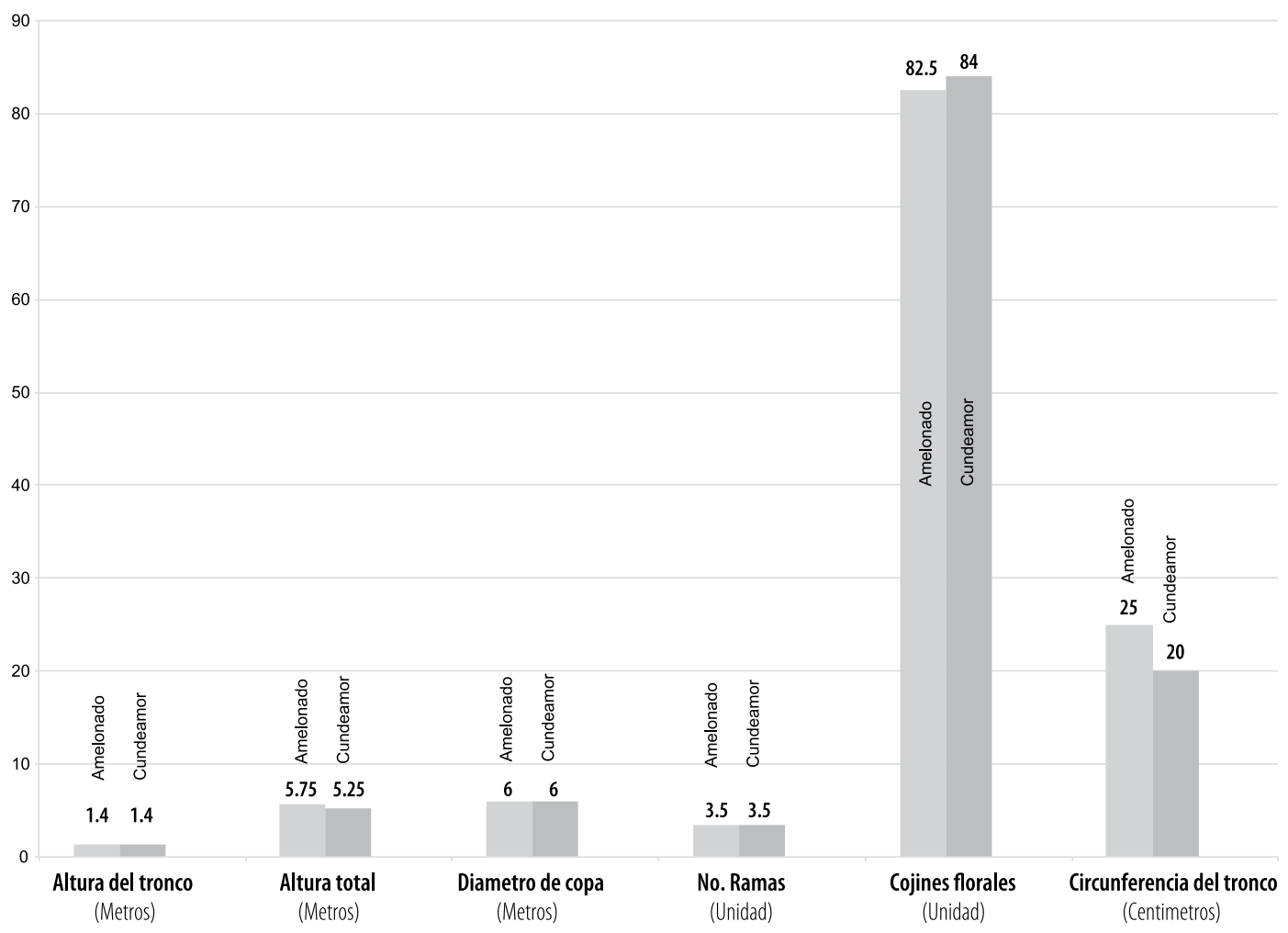

Figura No.5. Descriptores morfológicos de árboles seleccionados en la comunidad Zopilote.

En la comunidad de el Zopilote que pertenece al municipio de Rosita, se evaluaron 2 árboles con frutos de forma amelonados, 2 con frutos cundeamor. Los datos morfológicos de altura del tronco a la primera horqueta fueron de 1.3 - 1.5 metros, la altura total de los árboles fue de 5.0 a 6.0 metros, el diámetro de copa de 6.0 metros, número promedio de ramas de 3.0 - 4.0, los cojines florales oscilaron desde 80 como mínimo hasta 88 , presentado por los tipos amelonados, la circunferencia del tronco osciló entre 20 a 25 centímetros. 


\section{Comportamiento productivo de árboles de cacao seleccionados}

Para medir la capacidad productiva de los árboles seleccionados se determinaron criterios específicos de las "plantas madre", como: tolerancia a plagas y enfermedades, observar árboles con escasa incidencia de Moniliasis, Phytoptora, Mal del machete, Cherelle wild. Buena producción: más de 50 frutos sanos en el ciclo. Los rangos que se evaluaron fueron tamaño de los frutos por árbol, índice de mazorcas, índice de semillas, producción de árbol por año.

Cuadro No. 2. Comportamiento productivos de árboles de cacao en las comunidades de El Carao, Floripón, El Guayabo, Santa Rita y Zopilote

\begin{tabular}{|c|c|c|c|c|c|c|c|c|c|c|}
\hline Tipo Angoleta & \multicolumn{10}{|c|}{ Tipo Amelonado } \\
\hline $\begin{array}{l}\text { Indicadores } \\
\text { productivos }\end{array}$ & $\frac{8}{\frac{\pi}{0}}$ & 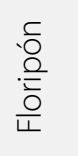 & $\frac{O}{\frac{0}{0}}$ & 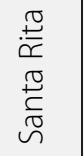 & $\frac{\stackrel{0}{\circ}}{\frac{0}{\bar{O}}}$ & $\frac{8}{\frac{0}{0}}$ & $\begin{array}{l}\stackrel{ }{\circ} \\
\stackrel{ }{\frac{0}{0}} \\
\text { 은 }\end{array}$ & 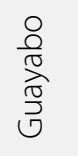 & 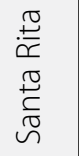 & $\frac{\stackrel{\Perp}{0}}{\frac{\bar{a}}{\circ}}$ \\
\hline Numero de árboles & 21 & 2 & 2 & 5 & 0 & 25 & 5 & 4 & 0 & 2 \\
\hline $\begin{array}{l}\text { Prom. frutos por } \\
\text { árbol }\end{array}$ & 65 & 69.5 & 56 & 72 & 0 & 65 & 68 & 71 & 0 & 59 \\
\hline $\begin{array}{l}\text { Prom. Índice de } \\
\text { mazorcas }\end{array}$ & 17.9 & 16 & 19.7 & 18.6 & O & 18 & 17 & 18 & 0 & 18.3 \\
\hline $\begin{array}{l}\text { Prom. Índice de } \\
\text { semillas }\end{array}$ & 1.4 & 1.4 & 1.39 & 1.36 & O & 1.36 & 1.45 & 1.35 & 0 & 1.345 \\
\hline $\begin{array}{l}\text { Prom. Produc. por } \\
\text { árbol }\end{array}$ & 3.71 & 4.6 & 3.105 & 3.82 & O & 3.42 & 3.98 & 3.18 & 0 & 3.18 \\
\hline Tipo cundeamor & \multicolumn{10}{|c|}{ Tipo calabacín } \\
\hline Numero de árboles & 3 & 2 & 3 & 2 & 1 & 1 & 0 & 0 & 0 & $\mathrm{O}$ \\
\hline $\begin{array}{l}\text { Promedio frutos por } \\
\text { árbol }\end{array}$ & 83 & 64 & 63 & 60 & 55 & 70 & & & & \\
\hline $\begin{array}{l}\text { Promedio ind. } \\
\text { Mazorcas }\end{array}$ & 19 & 17.5 & 18.5 & 18 & 18.5 & 22 & & & & \\
\hline $\begin{array}{l}\text { Prom. Índice de } \\
\text { semillas }\end{array}$ & 1.36 & 1.5 & 1.36 & 1.32 & 1.32 & 1.32 & & & & \\
\hline $\begin{array}{l}\text { Prom. Produc. por } \\
\text { árbol }\end{array}$ & 4.67 & 3.8 & 3.845 & 4 & 3.10 & 04.8 & & & & \\
\hline
\end{tabular}

Para la evaluación de los índices productivos representados en el cuadro dos, se analizaron cuatro indicadores: mazorcas por árbol, índice de mazorcas, índice de semillas y producción de árbol por año, se evaluaron cuatro tipos de cacao por comunidad con los siguientes resultados: 
Frutos por árbol para el tipo angoleta: El Carao, 21 árboles con producciones promedios de 65 frutos. En Floripón, 2 árboles con producciones promedios de 69.5 frutos. En el Guayabo, 2 árboles con producciones promedios de 56 frutos. En Santa Rita, 5 árboles con producciones promedios de 72 frutos.

El índice de mazorcas por kilogramo en la comunidad. El Carao promedió 18, en El Floripón 16, en El Guayabo 19.7, en Santa Rita 18.6. El índice de semilla en todas las comunidades osciló entre 1.3 a 1.55 gramos. La producción por árbol en el ciclo productivo de junio del 2009 a junio del 2010, en la comunidad el Carao promedió 3.71 kg, En Floripón 4.595 kg. En el Guayabo 3.105 kg y en Santa Rita 3.816 kg.

\section{Tipo amelonado}

Frutos por árbol. En el Carao se evaluaron 25 árboles con promedios de 65 frutos por año. En Floripón 5 árboles con promedios de 68 frutos por año. En el Guayabo 4 árboles con promedios de 71 frutos por año. En el Zopilote 2 árboles promedios de 59 frutos por año. El índice de mazorcas por kilogramo en la comunidad el Carao promedió 18 frutos, en el Floripón 17 frutos, en el Guayabo 18 frutos, Zopilote 18.3 frutos por kilogramo. El índice de semilla en la comunidad el Carao dio resultados promedios de 1.366 gramos, Floripón 1.44 gramos, Guayabo 1.35 gramos, Zopilote 1.345 gramos. La producción por árbol en el Carao promedió $3.421 \mathrm{~kg}$. En el Floripón $3.979 \mathrm{~kg}$. En el Guayabo $3.18 \mathrm{~kg}$. En El Zopilote $3.18 \mathrm{~kg}$.

\section{Tipo cundeamor}

Frutos por año. En el Carao se evaluaron 3 árboles con promedios de 83 frutos. En Floripón 2 árboles y promedios de 64 frutos. En el Guayabo 3 árboles con promedios de 63 frutos. En el Zopilote 1 árbol con promedios de 70 frutos. En Santa Rita 2 árboles con 55 frutos por año. El índice de mazorcas por kilogramo en la comunidad el Carao promedió 19 frutos, en el Floripón 17.5. Guayabo 18.5 frutos. Santa Rita 18.5 frutos; en Zopilote, 23 máximo, 22 frutos por kilogramo. El índice de semilla en la comunidad el Carao dio promedió 1.366 gramos, Floripón 1.36, Santa Rita 1.32. Zopilote 1.32. La producción por árbol en el Carao promedió $4.673 \mathrm{~kg}$, en el Floripón $3.8 \mathrm{~kg}$, en el Guayabo 3.845 kg, en Santa Rita 4 kg, en el Zopilote 3.105 kg.

\section{Tipo calabacín}

En el Carao se evaluó 1 árbol con producciones de 70 mazorcas por año. El índice de mazorcas por kilogramo promedió 22. El índice de semilla fue de 1.32 gramos. La producción por árbol promedió $4.805 \mathrm{~kg}$. 


\section{Plagas y enfermedades en árboles seleccionados en el Carao, Floripón, el Guayabo, Santa Rita y Zopilote}

A través del levantamiento técnico que se realizó en las diferentes comunidades se pudo apreciar la incidencia de plagas y enfermedades las que tuvieron algún grado de afectación según el tipo de árboles muestreados. A continuación se describe el grado de afectación por tipos de árboles de cacao muestreados y por comunidad.

\section{Tipo angoleta}

En el Carao se muestrearon 21 árboles con producción de 1,876 frutos, con afectación de 300 frutos en el año; en Floripón se muestreó un árbol con producción de 39 frutos, uno afectado; en el Guayabo se muestrearon dos árboles con producción de 168 frutos, 12 afectados; en Santa Rita se muestrearon cinco árboles con producciones de 455 frutos, 93 afectados. La afectación porcentual por plagas y enfermedades en este tipo de cacao fue: El Carao, $18.33 \%$ por Monilia, $10.66 \%$ por mazorca negra, $8 \%$ por Cherelle wild, 63\% por gusanos. Floripón 2.6\% por mazorca negra. El Guayabo, 8.33\% por Monilia, 50\% por Cherelle wild, $41.6 \%$ por gusanos. Santa Rita, $96.77 \%$ por Cherelle wild, y $3.33 \%$ por gusanos.

\section{Tipo amelonado}

En el Carao se muestrearon 25 árboles con producción de 1,566 frutos, hubo una afectación de 223 frutos, En el Floripón se muestrearon 5 árboles los que produjeron 557 frutos, de estos 49 fueron afectados. En el Guayabo se muestrearon 5 árboles los que produjeron 388 frutos, 36 fueron afectados. En el Zopilote se muestrearon dos árboles con producción de 129 frutos, 11 afectaciones. La afectación por plagas y enfermedades fue: El Carao, 2.24 \% por Moniliasis, 4\% por mazorca negra, $40.3 \%$ por Cherelle wild y 53.4\% por gusanos. Floripón, $2 \%$ por Moniliasis, $2 \%$ por mazorca negra, $30.6 \%$ por Cherelle wild y $65 \%$ por gusanos. El Guayabo, $19 \%$ Moniliasis, $16.6 \%$ por mazorca negra, 30.5\% por Cherelle wild, 33.3\% por gusanos. Rosa Grande, 100\% afectaciones por gusanos. El Zopilote, $\mathbf{2 7 . 2} \%$ Cherelle wild y $72.8 \%$ por gusanos.

\section{Tipo cundeamor}

En todas las comunidades en estudio se muestrearon 7 árboles los que produjeron 505 frutos de los cuales 20 fueron atacados por plagas y enfermedades, sobresaliendo la afectación por gusanos y abortos sólo 1 fruto fue por mazorca negra. De toda la producción se afectó el 4\%, siendo la de mayor importancia la muerte prematura de frutos y los gusanos. 


\section{Tipo calabacín}

En la comunidad El Carao se muestreó un árbol el que produjo 73 frutos con afectaciones de 3 frutos por gusanos. El porcentaje de afectación por plagas fue de $4.1 \%$. Este tipo de cacao no es de importancia económica para los productores ya que produce frutos pequeños con almendras menores de 1.3 gramos de peso, lo que ocasiona una fermentación no homogénea al mezclarse con otros tipos de cacao.

\section{Conclusiones}

Las condiciones agroecológicas de las plantaciones en estudio son similares, sólo difieren en altura sobre el nivel del mar, desde $55-100 \mathrm{msnm}, 100-200 \mathrm{msnm}$ y 400 $-525 \mathrm{msnm}$.

El número de frutos promedios por tipos de cacao y por año fue: tipo angoleta: 63 en el Carao, 70 en Floripón, 65 en el Guayabo y 72 en Santa Rita. Tipo amelonado: 65 en el Carao, 68 en Floripón, 55 en el Guayabo y 52 en el Zopilote. Tipo cundeamor: 83 en el Carao, 64 en Floripón, 63 en el Guayabo. Tipo calabacín: 70 en el Carao.

Los árboles tipo angoleta, amelonado y cundeamor presentaron índices promedios de 16 a 19 mazorcas por kilogramo, los tipos calabacín 22 mazorcas por Kilogramo. Los índices de semillas oscilaron entre 1.3 a 1.43 en los tipos angoletas, 1.34 a 1.45 en los tipos amelonados, 1.32 a 1.5 en los tipos cundeamor y 1.32 en los tipos calabacín. La producción promedio por árbol osciló entre 3.11 a 4.6 kilogramos por árbol en los tipos angoletas, 3.1 a 4.00 kilogramos en los amelonados, 3.11 a 4.7 en los cundeamor y o.6 kilogramos en los calabacín.

La mayoría de los árboles evaluados presentaron afectaciones por plagas y enfermedades como: Moniliasis, mazorca negra, Cherelle wild y gusanos en los frutos, así como afectaciones por tandas en ramas. La enfermedad que tiene más incidencia en las plantaciones es la Moniliasis y entre las plagas que más afectan los frutos sobresalen los gusanos comedores de frutos. 


\section{Lista de referencias}

Sánchez A. (1990). Manual para la educación agropecuaria de cultivo de plantación. México: Trillas, S.A.C.V

Calderón E. (1935). Manual del cacao para agricultores San José C.R. Universidad Estatal a Distancia San José, Costa Rica, 1987. P. 9-11.

Cerda R. (s.f) CaTIE. Selección de árboles superiores de cacaoen la finca de familia Cacaoteras. P. 1-3.

Bacardit R. (2001). Producción de plantas de cacao por injerto. www.zoetecnocampo. com/forodocs/.../o00002 Recuperado el 13 Agosto del 2009. P.

Avilés G. (2008). Evaluación agronómico de 12 clones y 15 híbridos promisorios de cacao (Theobroma cacao L.), en condiciones de los Guatuzos, San Carlo, río San Juan. Nicaragua, en el período del 2008 al 2010. www.inta.gob.ni/.../aet-pro-cloneshibridos-cacao-losguatuzos.doc. Recuperado el 12 Agosto del 2009. P. 2.

PROAMAZONIA. (2004). Manual del cultivo de cacao cecoeco. CATIE.ac.cr/descargas/ Manual_Cacao-Peru.pdf. Recuperado el 13 Agosto del 2009. P. 9-13- 55-57.

Enríquez G, (s.f). Tecnología de cacao. www.infoagro.go.cr/.../cacao/plagasyenfermedades.htm. Recuperado 12 Agosto del 2009. P. 5-8. 